\title{
Modelos de regressão polinomiais na otimização de manejos para a máxima produtividade industrial com qualidade química em grãos de aveia
}

\author{
Vanessa Pansera ${ }^{1}$ \\ DCEENG/UNIJUÍ, Ijuí, RS \\ Rúbia Diana Mantai ${ }^{2}$ \\ DCEENG/UNIJUí, Ijuí, RS \\ José Antonio Gonzalez da Silva ${ }^{3}$ \\ DEAG/UNIJUí, Ijuí, RS \\ Roberto Carbonera ${ }^{4}$ \\ DEAG/UNIJUÍ, Ijuí, RS \\ Adriana Roselia Kraisig ${ }^{5}$ \\ DCEENG/UNIJUÍ, Ijuí, RS \\ Odenis Alessi ${ }^{6}$ \\ DCEENG/UNIJUÍ, Ijuí, RS \\ Felipe Uhde Porazzi ${ }^{7}$ \\ DEAG/UNIJUÍ, Ijuí, RS \\ Cristhian Milbradt Babeski ${ }^{8}$ \\ DEAG/UNIJUÍ, Ijuí, RS
}

Resumo. O uso de modelos de regressão na agricultura permite a análise e otimização de manejos. A aveia é um cereal que possui diversas qualidades nutricionais para alimentação humana e é dependente de nitrogênio $(\mathrm{N})$ para garantir produtividade com qualidade. O objetivo deste estudo é empregar modelos de regressão polinomiais na otimização do uso de nitrogênio no cultivo da aveia, maximizando a produtividade industrial com previsibilidade da expressão de componentes relacionados à qualidade química de grãos. Para isto, foi realizado um experimento em condições reais de cultivo da aveia, em delineamento de blocos casualizados com quatro repetições, em um fatorial $4 \times 6$ ( 4 doses de nitrogênio e 6 anos agrícolas). Foram realizadas análises de regressão linear e quadrática. O nitrogênio evidenciou eficiência no incremento de $3,3 \mathrm{~kg} \mathrm{ha}^{-1}$ de produtividade industrial (PI) e de $0,10 \mathrm{~g} \mathrm{~kg}^{-1}$ de grãos de proteína total (PT), a cada quilograma de $\mathrm{N}$ aumentado por hectare, independente de ano agrícola. Porém, quanto à fibra total (FT), houve um decremento de $0,05 \mathrm{~g} \mathrm{~kg}^{-1}$, nas mesmas condições. A dose mais ajustada de $\mathrm{N}$ ocorre em torno de $83 \mathrm{~kg} \mathrm{ha}^{-1}$, independente de ano agrícola, garantindo elevada produtividade industrial de grãos de aveia, com componentes de qualidade química estimados em $109 \mathrm{~g} \mathrm{~kg}^{-1}$ de PT e $125 \mathrm{~g} \mathrm{~kg}^{-1}$ de FT.

Palavras-chave. Modelagem Matemática, Modelos Estatísticos, Sustentabilidade, Qualidade Nutricional, Avena sativa

\footnotetext{
${ }^{1}$ Bolsista Capes/Brasil, vpansera@hotmail.com

${ }^{2}$ rdmantai@yahoo.com.br

3 jagsfaem@yahoo.com.br

${ }^{4}$ carbonera@unijui.edu.br

${ }^{5}$ maryshelei@yahoo.com.br

${ }^{6}$ odenisalessi@hotmail.com

7 felipe.uhde@hotmail.com

8 cristhiancmb@hotmail.com
} 


\section{Introdução}

A modelagem matemática é uma importante ferramenta para a análise de fenômenos reais $[2,8]$. Na agricultura, o uso de regressões lineares e quadráticas permite verificar o desempenho de manejos, auxiliando na determinação de práticas mais sustentáveis $[1,7]$. Através da análise de coeficientes da regressão linear, é possível verificar a taxa de variação de uma variável dependente em relação a uma variável controlada, caracterizando a eficiência agronômica [5,6]. Enquanto que na regressão quadrática, por meio da identificação do ponto ótimo da função, pode-se indicar a máxima eficiência técnica de um manejo [6].

A aveia é uma cultura agrícola que pode ter seu cultivo beneficiado devido à utilização da modelagem matemática. Este cereal possui características químicas importantes, destacando-se a presença de proteínas e de fibras, que podem reduzir os teores de colesterol LDL do sangue e o risco de doenças cardiovasculares $[1,2,4]$. Para o processamento industrial da aveia na obtenção de alimentos, é utilizada apenas a cariopse do grão, ou seja, somente seu interior, sem a casca, considerando ainda os grãos com espessura acima de $2 \mathrm{~mm}$. Estas características definem a produtividade industrial, variável importante a ser considerada no cultivo da aveia [2].

Um nutriente essencial a ser aplicado durante o desenvolvimento da cultura é o nitrogênio $(\mathrm{N})$, que influencia diretamente a produtividade de grãos, a produtividade industrial e composição química dos grãos. Entretanto, se aplicado em demasia pode causar a contaminação do solo, e se aplicado em quantidade inferior à necessária, pode prejudicar o desenvolvimento da planta $[1,5,6]$.

$\mathrm{O}$ uso de regressões pode auxiliar na determinação da dose mais ajustada de nitrogênio à máxima produtividade industrial e química de grãos de aveia. O objetivo deste estudo é empregar modelos de regressão polinomiais na otimização do uso de nitrogênio no cultivo da aveia, maximizando a produtividade industrial com previsibilidade da expressão de componentes relacionados à qualidade química de grãos.

\section{Metodologia}

Para o desenvolvimento deste estudo, inicialmente foi realizado um experimento em condições reais de cultivo. Este ocorreu no Instituto Regional de Desenvolvimento Rural (IRDeR) pertencente à Universidade Regional do Noroeste do Estado do Rio Grande do Sul (UNIJUÍ) em Augusto Pestana (RS). Foram utilizadas duas cultivares de aveia branca (Brisasul e Barbarasul), sendo consideradas as médias de seus resultados. O delineamento experimental foi de blocos casualizados com quatro repetições, em um fatorial $4 \times 6$, com 4 doses de nitrogênio $\left(0,30,60\right.$ e $\left.120 \mathrm{~kg} \mathrm{ha}^{-1}\right)$ e 6 anos agrícolas (2011 a 2016), no sistema de sucessão soja/aveia. As parcelas experimentais foram constituídas de $5 \mathrm{~m}^{2}$, com posterior conversão dos dados para hectare. As variáveis respostas foram obtidas em laboratório, com foco principal para a qualidade industrial e química de grãos, considerando a produtividade industrial (PI), a proteína total (PT) e a fibra total (FT).

A produtividade industrial foi calculada por

$$
P I=P G \cdot \frac{N G>2 m m}{100} \cdot I D
$$

em que $P G$ é a produtividade de grãos, $N G>2 m m$ é o número de grãos com espessura maior que $2 \mathrm{~mm}$ considerando um conjunto de 100 grãos e $I D$ é o indíce de descasque, dado por

$$
I D=\frac{M_{C}}{M_{G}}
$$

em que $M_{C}$ é a massa da cariopse de 50 grãos e $M_{G}$ é a massa de 50 grãos [2]. 
Como os anos agrícolas possuem diferenças quanto às condições meteorológicas, influenciando no cultivo da aveia [5,6], optou-se por classificá-los em favoráveis, intermediários ou desfavoráveis à produtividade de grãos $(\mathrm{PG})$. Considerou-se os valores de precipitação e temperaturas ocorridos durante o ciclo da cultura, relacionando à média histórica de precipitação dos últimos 25 anos e considerando a PG obtida em cada ano, visto que as cultivares receberam adubação nitrogenada

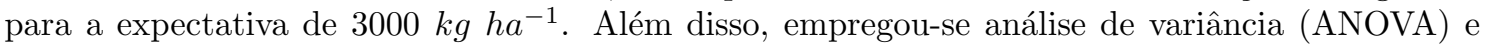
o modelo de Scott-Knott para auxiliar na classificação $[1,3,5,6]$. Em todos os testes estatísticos realizados neste estudo foi considerado nível de significância de $5 \%$ de probabilidade de erro.

$\mathrm{Na}$ análise dos dados, para verificar a existência de diferença significativa entre os tratamentos e se há interação entre os mesmos sobre as variáveis respostas, os dados foram submetidos à ANOVA $[1,6]$. Em seguida foi empregado o modelo de Scott-Knott $[3,5]$. Neste estudo optou-se por desenvolver análise de regressão considerando apenas o tratamento relativo ao nitrogênio, sem efeitos de anos agrícolas, de interação e de blocos. Visto que os anos de cultivo não são fatores controlados, optou-se por classificá-los e realizar as análises individualmente em cada ano, com modelos simplificados. Justifica-se esta metodologia pela intenção de analisar o efeito direto do nitrogênio em relação às variáveis respostas, conforme o cenário proporcionado pelo ano agrícola.

A eficiência agronômica do nitrogênio, em cada ano de cultivo, sobre a PI, PT e FT, foi determinada através da análise do coeficiente angular das regressões lineares obtidas com o modelo

$$
y=\beta_{0}+\beta_{1} x+\varepsilon
$$

em que $y$ é a variável resposta (PI, PT ou FT), $x$ é a variável independente (que está relacionada ao nitrogênio), $\beta_{0}$ é o coeficiente linear, $\beta_{1}$ é o coeficiente angular e $\varepsilon$ é o erro aleatório $[3,6]$.

A máxima eficiência técnica do uso de nitrogênio, a fim de maximizar a produtividade industrial, foi obtida considerando o modelo de regressão quadrático e seu ponto de máximo, dados por

$$
\begin{gathered}
y=\beta_{0}+\beta_{1} x+\beta_{2} x^{2}+\varepsilon \\
x_{M E T}=\frac{-\beta_{1}}{2 \beta_{2}}
\end{gathered}
$$

em que $y$ e $x$ possuem as mesmas definições da equação (3), $x_{M E T}$ é a máxima eficiência técnica, $\beta_{0}, \beta_{1}$ e $\beta_{2}$ são os coeficientes da regressão e $\varepsilon$ é o erro aleatório $[3,6]$.

Com a obtenção da máxima eficiência técnica do nitrogênio em cada ano agrícola, foi determinado um valor de $\mathrm{N}$ ideal, independente de ano. Na sequência, foram simulados os níveis de proteína e fibra totais em função deste valor.

\section{Resultados e Discussões}

Ao ser aplicada a ANOVA sobre os anos de cultivo, percebeu-se diferença estatística na resposta da produtividade de grãos de aveia. Em 2011 e 2013 houve chuvas com boa distribuição ao longo do ciclo. Em 2011, a precipitação alcançou níveis próximos à média histórica e em 2013 houve precipitação abaixo da média histórica. Entretanto, a boa distribuição de umidade e as temperaturas mais amenas, em ambos os anos, contribuíram para o adequado desenvolvimento da planta. As produtividades médias de grãos nestes anos foram de, respectivamente, 3686 e $3731 \mathrm{~kg}$ $h a^{-1}$, ou seja, acima da expectativa de $3000 \mathrm{~kg} \mathrm{ha}^{-1}$, com médias pelo modelo de Scott-Knott na categoria " $a$ ", ou seja, foram as médias de PG mais expressivas a serem observadas. Isto caracterizou 2011 e 2013 como anos favoráveis (AF) à produtividade de grãos de aveia.

Em 2012, no início do ciclo ocorreram reduzidas precipitações e elevadas temperaturas, enquanto em 2014 os primeiros dias do ciclo foram marcados por um volume de chuvas expressivos 
acima da média histórica, também com temperaturas elevadas. Estas condições extremas possivelmente acarretaram no mal aproveitamento do nitrogênio. Além disso, ao final do ciclo em ambos os casos, as chuvas foram mais presentes e com maior valor acumulado, atrasando a colheita de grãos. Nestes anos a produtividade de grãos foi inferior à expectativa desejada, com produtividades de 2378 e $2181 \mathrm{~kg} \mathrm{ha}^{-1}$, respectivamente, apresentando baixo desempenho pelo modelo de Scott-Knott, em categorias "c"e "d". Estes fatores justificam a classificação dos anos 2012 e 2014 como anos desfavoráveis (AD) à produtividade de grãos de aveia.

Em 2015, a precipitação acumulada foi próxima à média observada de 25 anos. Condições adequadas de umidade do solo beneficiaram a aplicação de nitrogênio, contudo, houve altas temperaturas durante o período de polinização, momento que é sensível ao estresse hídrico e altas temperaturas. O ano de 2016 foi marcado por baixas precipitações, principalmente no período de enchimento de grãos, com fortes chuvas na fase final do ciclo, momento em que a produtividade de grãos já está decidida, porém a qualidade do grão ainda é influenciada. As temperaturas se mostraram adequadas e estáveis. As produtividades médias observadas nestes anos foram de 3451

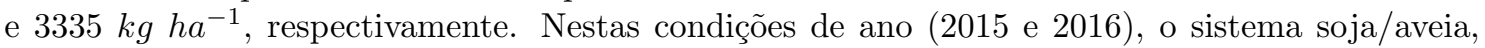
de baixa relação carbono-nitrogênio $(\mathrm{C} / \mathrm{N})$, possivelmente pelo aporte do N-residual, fez com que a produtividade de grãos superasse a expectativa de $3000 \mathrm{~kg} \mathrm{ha}^{-1}$, fato que não ocorreria em outros sistemas de cultivo de alta relação $\mathrm{C} / \mathrm{N}$. Pelo modelo de Scott-Knott, as médias receberam agrupamento "b", ou seja, com desempenho inferior aos anos com características favoráveis. Por estes fatos, classifica-se os anos de 2015 e 2016 como intermediários (AI) à produtividade de grãos.

Após a obtenção das variáveis relativas à produtividade industrial e química ao longo dos anos agrícolas em função das diferentes doses de aplicação de nitrogênio, os dados foram submetidos à ANOVA. Foram constatadas diferenças significativas entre os anos de cultivo e as doses de nitrogênio, bem como a existência de interação entre os tratamentos. Assim, foi empregado o modelo de comparação de médias de Scott-Knott na identificação dos agrupamentos existentes entre as médias obtidas no experimento. Conjuntamente a isto, foi realizada análise de regressão linear com a finalidade de determinar a eficiência agronômica do nitrogênio ao longo dos anos.

Na Tabela 1, considerando os resultados do modelo de Scott-Knott, quanto à produtividade industrial, percebe-se bons resultados para os anos de 2011, 2012, 2013 e 2015. Em 2011 e 2012 são encontradas médias similares estatisticamente, embora um ano seja considerado favorável à produtividade de grãos e o outro seja classificado como desfavorável. Além disso, observando o desempenho industrial das cultivares em anos classificados como intermediários, há tanto resultados positivos, como negativos. Isto aponta para o fato de que uma elevada produtividade de grãos não necessariamente está relacionada a uma alta produtividade industrial, isto porque a quantidade de grãos com espessura maior que $2 \mathrm{~mm}$ e o índice de descasque influenciam fortemente na qualidade industrial. Por outro lado, quando observado o agrupamento de Scott-Knott para as médias de PI em função da quantidade de nitrogênio aplicada, percebe-se bons resultados no desempenho das cultivares quando há a aplicação de doses maiores, inclusive com mesmo desempenho estatístico

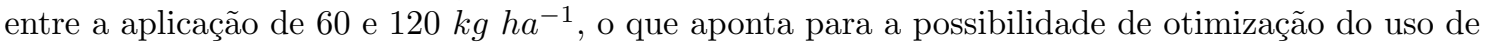
nitrogênio quando utilizada a dose de $60 \mathrm{~kg} \mathrm{ha} \mathrm{h}^{-1}$.

Quanto à proteína total, é possível observar através dos agrupamentos do modelo de ScottKnott bons resultados independente de ano agrícola, porém com melhora significativa à medida que aumentam as doses de nitrogênio aplicadas. Entretanto, a fibra total tem seus valores médios reduzidos quando há o aumento na dose $\mathrm{N}$, além de apresentar valores mais elevados em anos desfavoráveis à produtividade de grãos e mais reduzidos em anos favoráveis. Isto evidencia a necessidade de otimizar o uso de nitrogênio, com objetivo de maximizar a produtividade industrial e manter equilibrados os níveis de proteína e fibra nos grãos.

Ainda na Tabela 1, na análise das regressões lineares, observou-se eficiência agronômica significativa do $\mathrm{N}$ nos anos de 2012, 2013 e 2014 para a produtividade industrial, com aumento 


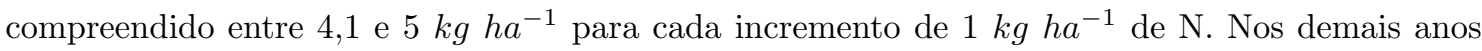
também observou-se incremento da produtividade quando aumentada a dose do nutriente, com

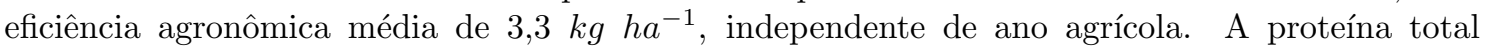
também apresentou crescimento em seus valores em função do aumento da dose de nitrogênio, com uma eficiência agronômica média de $0,10 \mathrm{~g}$ por quilograma de grãos a cada quilograma de $\mathrm{N}$ aplicado por hectare. Os aumentos mais significativos ocorreram nos anos de 2011, 2012, 2013 e 2016. Quanto à fibra total, percebeu-se decremento em seus valores à medida que é aumentada a dose de $\mathrm{N}$, com redução média de $0,05 \mathrm{~g} \mathrm{~kg}^{-1}$ para cada quilograma de $\mathrm{N}$ aplicado por hectare.

Tabela 1: Equações da eficiência agronômica e valores médios da produtividade industrial e qualidade química de grãos de aveia em função de diferentes doses de nitrogênio em distintos anos de cultivo.

\begin{tabular}{|c|c|c|c|c|c|c|c|c|c|}
\hline \multirow[t]{2}{*}{$y$} & \multirow[t]{2}{*}{ Ano } & \multicolumn{4}{|c|}{ Valores médios/ dose de $\mathrm{N}\left(k g h a^{-1}\right)$} & \multirow[t]{2}{*}{$\bar{y}$} & \multirow{2}{*}{$\begin{array}{c}\text { Equação } \\
y=b_{0}+b_{1} x\end{array}$} & \multirow{2}{*}{$\begin{array}{c}\mathrm{P} \\
\left(b_{i} x\right)\end{array}$} & \multirow{2}{*}{$\begin{array}{l}R^{2} \\
(\%)\end{array}$} \\
\hline & & 0 & 30 & 60 & 120 & & & & \\
\hline \multirow{7}{*}{$\begin{array}{c}\mathrm{PI} \\
\left(k g h a^{-1}\right)\end{array}$} & $2011(\mathrm{AF})$ & 1109 & 1578 & 1698 & 1560 & $1486 \mathrm{~b}$ & $1324+3,1 x$ & $n s$ & 37 \\
\hline & 2012 (AD) & 1037 & 1440 & 1665 & 1689 & $1458 \mathrm{~b}$ & $1194+5,0 x$ & $*$ & 73 \\
\hline & $2013(\mathrm{AF})$ & 1504 & 1810 & 2169 & 2108 & $1898 \mathrm{a}$ & $1639+4,9 x$ & $*$ & 68 \\
\hline & 2014 (AD) & 891 & 1100 & 1294 & 1393 & $1170 \mathrm{c}$ & $955+4,1 x$ & $*$ & 89 \\
\hline & $2015(\mathrm{AI})$ & 1098 & 1453 & 1701 & 1442 & $1424 \mathrm{~b}$ & $1292+2,5 x$ & $n s$ & 27 \\
\hline & $2016(\mathrm{AI})$ & 315 & 403 & 424 & 355 & $374 \mathrm{~d}$ & $364+0,2 x$ & $n s$ & 4 \\
\hline & $\bar{x}$ & $992 \mathrm{C}$ & $1297 \mathrm{~B}$ & $1492 \mathrm{~A}$ & $1425 \mathrm{~A}$ & 1302 & $1128+3,3 x$ & * & 59 \\
\hline \multirow{7}{*}{$\begin{array}{c}\mathrm{PT} \\
\left(g k g^{-1}\right)\end{array}$} & 2011 (AF) & 104,9 & 106,8 & 111,8 & 118,6 & $110,5 \mathrm{a}$ & $104,3+0,12 x$ & * & 98 \\
\hline & 2012 (AD) & 103,1 & 105,9 & 110,1 & 119,5 & $110,0 \mathrm{a}$ & $102,4+0,14 x$ & $*$ & 99 \\
\hline & $2013(\mathrm{AF})$ & 98,1 & 102,3 & 102,5 & 107,0 & $102,5 \mathrm{~b}$ & $98,9+0,07 x$ & $*$ & 94 \\
\hline & 2014 (AD) & 98,5 & 101,1 & 102,9 & 105,9 & $102,1 \mathrm{~b}$ & $98,9+0,06 x$ & $n s$ & 98 \\
\hline & $2015(\mathrm{AI})$ & 107,8 & 108,5 & 109,5 & 112,0 & $109,5 \mathrm{a}$ & $107,6+0,03 x$ & $n s$ & 98 \\
\hline & $2016(\mathrm{AI})$ & 94,8 & 97,1 & 102,4 & 115,0 & $102,3 \mathrm{~b}$ & $93,2+0,17 x$ & $*$ & 97 \\
\hline & $\bar{x}$ & $101,2 \mathrm{D}$ & $103,6 \mathrm{C}$ & $106,5 \mathrm{~B}$ & $113,0 \mathrm{~A}$ & 106,1 & $100,9+0,10 x$ & * & 99 \\
\hline \multirow{7}{*}{$\begin{array}{c}\mathrm{FT} \\
\left(g k g^{-1}\right)\end{array}$} & $2011(\mathrm{AF})$ & 128,5 & 123,4 & 121,9 & 120,8 & $123,6 \mathrm{~d}$ & $126,6-0,06 x$ & * & $\overline{76}$ \\
\hline & 2012 (AD) & 136,9 & 135,8 & 132,6 & 131,5 & $134,2 \mathrm{a}$ & $136,6-0,05 x$ & * & 90 \\
\hline & $2013(\mathrm{AF})$ & 118,9 & 117,6 & 116,9 & 115,3 & $117,2 \mathrm{e}$ & $118,7-0,03 x$ & $n s$ & 99 \\
\hline & 2014 (AD) & 133,3 & 132,3 & 130,2 & 128,5 & $131,1 \mathrm{~b}$ & $133,1-0,04 x$ & $*$ & 98 \\
\hline & $2015(\mathrm{AI})$ & 134,0 & 129,8 & 128,0 & 128,0 & $130,0 \mathrm{~b}$ & $132,2-0,04 x$ & $n s$ & 65 \\
\hline & $2016(\mathrm{AI})$ & 127,8 & 126,4 & 124,4 & 123,0 & $125,4 \mathrm{c}$ & $127,4-0,04 x$ & $n s$ & 94 \\
\hline & $\bar{x}$ & $129,9 \mathrm{~A}$ & $127,5 \mathrm{~B}$ & $125,7 \mathrm{C}$ & $124,5 \mathrm{D}$ & 126,9 & $129,2-0,05 x$ & $*$ & 91 \\
\hline
\end{tabular}

$\mathrm{N}=$ nitrogênio; $\mathrm{PI}=$ produtividade industrial; $\mathrm{PT}=$ proteína total; $\mathrm{FT}=$ fibra total; $\mathrm{AF}=$ ano favorável; $\mathrm{AD}=$ ano

desfavorável; $\mathrm{AI}=$ ano intermediário; $\bar{y}=$ média da variável $y ; P\left(b_{i} x\right)=$ probabilidade do parâmetro de inclinação da reta; ${ }^{*}=$ significativo a $5 \%$ de probabilidade de erro, pelo teste $t ;{ }^{n s}=$ não significativo a $5 \%$ de probabilidade de erro, pelo teste t; $R^{2}$ = coeficiente de determinação; Médias seguidas pelas mesmas letras minúsculas na coluna e maiúsculas na

linha, constituem grupo estatisticamente homogêneo pelo modelo de Scott-Knott a $5 \%$ de probabilidade de erro.

A regressão linear permite verificar a eficiência agronômica de manejos ao se analisar a taxa de variação de variáveis respostas em relação a variáveis controladas. Já o uso de regressão quadrática auxilia na obtenção da máxima eficiência técnica (MET) de manejos, através da verificação do ponto ótimo da função. Assim, na Figura 1 são apresentadas as regressões quadráticas obtidas da relação entre a produtividade industrial e doses de nitrogênio aplicadas, em cada ano de cultivo. Os valores encontrados para a máxima eficiência técnica do uso de nitrogênio, de 2011 até 2016, foram, respectivamente, de $76,87,89,112,76$ e $57 \mathrm{~kg} \mathrm{ha}^{-1}$, resultando nos respectivos valores de produtividade industrial: 1760, 1718, 2189, 1385, 1721 e $414 \mathrm{~kg} \mathrm{ha} \mathrm{H}^{-1}$.

Em anos desfavoráveis ao cultivo da aveia, como 2012 e 2014, houve maior dependência do uso de nitrogênio para a máxima eficiência. Enquanto em anos favoráveis, como 2011 e 2013, mesmo com menor uso de $\mathrm{N}$ obteve-se valores mais elevados de produtividade industrial. E em situações intermediárias, como as ocorridas em 2015 e 2016, houve reduzida necessidade de N para o ponto de máximo, porém, apresentando resultados bastante distintos. Em 2015 ocorreu uma produtividade 
industrial mais elevada que as obtidas em ambos os anos desfavoráveis e em 2016 ocorreu um valor de PI mais reduzido quando realizada a mesma comparação. Este fato vai ao encontro da análise realizada para 2016 durante a classificação dos anos agrícolas, em que constatou-se elevada precipitação ao final do ciclo que prejudicou a qualidade industrial dos grãos.

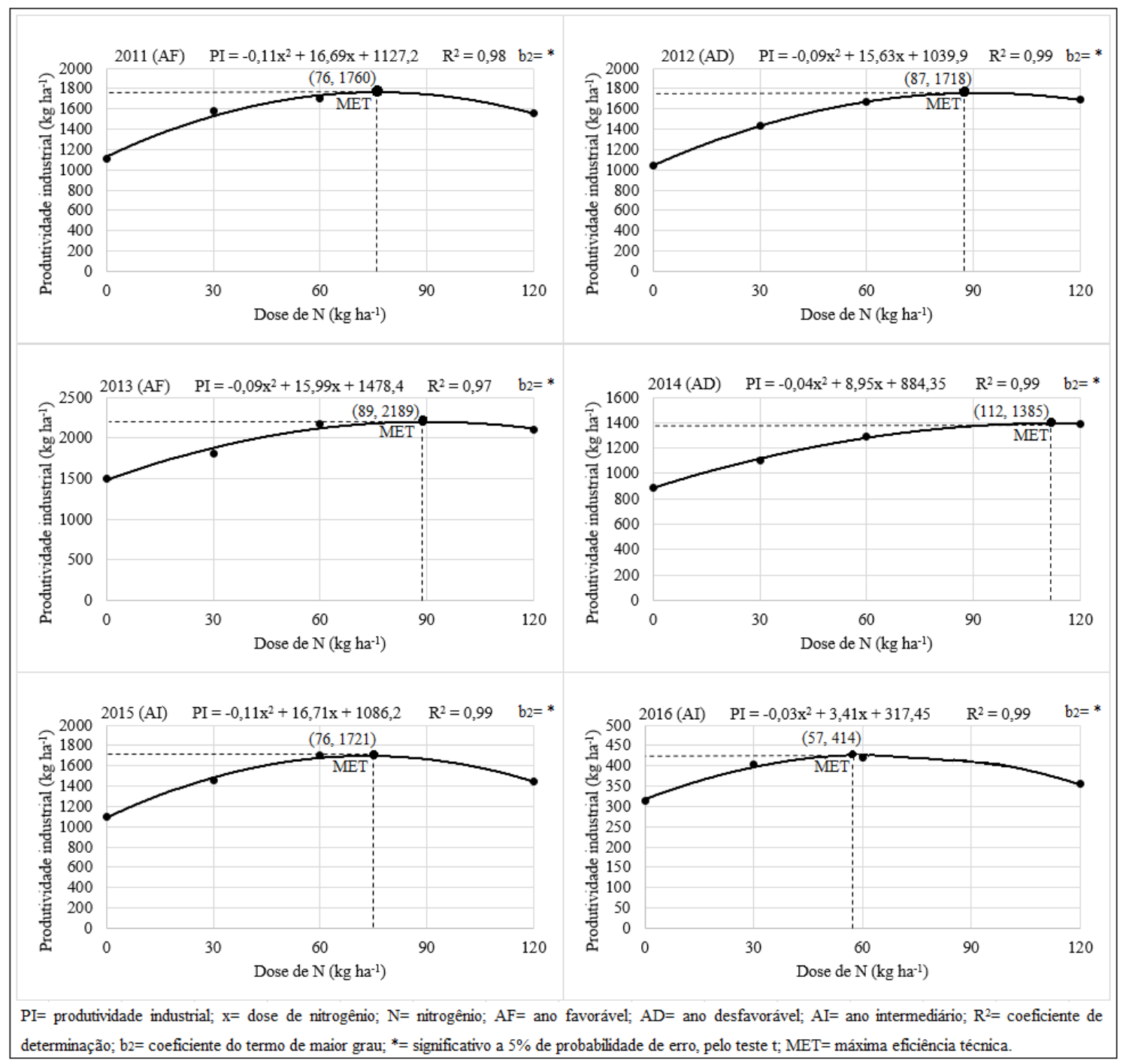

Figura 1: Máxima eficiência técnica do uso de nitrogênio sobre a produtividade industrial de aveia em diferentes anos de cultivo.

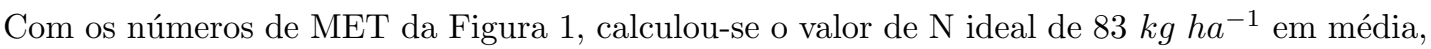
independente de ano agrícola, valor que está entre as doses de 60 e $120 \mathrm{~kg} \mathrm{ha}^{-1}$, observadas com mesmo agrupamento por Scott-Knott na Tabela 1 no efeito sobre a PI. Com este valor de N recomendado, simulou-se as quantidades de proteína e fibras totais, considerando as equações lineares médias (Tabela 1), também independentes de condições de ano. Para a proteína total obteve-se o valor de $109 \mathrm{~g} \mathrm{~kg}^{-1}$ e para a fibra total $125 \mathrm{~g} \mathrm{~kg}^{-1}$, quantidades próximas às médias correspondentes observadas na Tabela 1, que evidenciam bons níveis destas características químicas. 


\section{Conclusões}

As regressões lineares e quadráticas são uma importante ferramenta para a análise da eficiência e otimização do uso de nitrogênio no cultivo da aveia. Independente de condição de ano agrícola, é determinada a dose ideal de nitrogênio em torno de $83 \mathrm{~kg} \mathrm{ha}^{-1}$ para a maximização da produtividade industrial, com componentes de qualidade química estimados em $109 \mathrm{~g} \mathrm{~kg}^{-1}$ de proteína total e $125 \mathrm{~g} \mathrm{~kg}^{-1}$ de fibra total.

Em análises futuras, sugere-se a obtenção de modelos multivariados que descrevam a relação entre os dados, a fim de explicitar a interferência entre as variáveis de modo concomitante.

\section{Agradecimentos}

À CAPES, ao CNPq, à FAPERGS e à UNIJUÍ pelos recursos financeiros e estrutura física e material necessários para realização da pesquisa.

\section{Referências}

[1] Coelho, A. P., De Faria, R. T., Dalri, A. B., Palaretti, L. F. e Zanini, J. R. Clorofilômetro portátil como forma de manejo da irrigação e adubação nitrogenada em aveia-branca, Revista Brasileira de Agricultura Irrigada, 12:2542-2553, 2018. DOI: 10.7127/rbai.v12n200803.

[2] Crestani, M., De Carvalho, F. I. F., De Oliveira, A. C., Da Silva, J. A. G., Gutkoski, L. C., Sartori, J. F., Barbieri, R. L. e Baretta, D. Conteúdo de $\beta$-glucana em cultivares de aveia-branca cultivadas em diferentes ambientes, Pesquisa Agropecuária Brasileira, 45:261268, 2010. DOI: 10.1590/S0100-204X2010000300005.

[3] Cruz, C. D. Programa GENES: estatística experimental e matrizes. UFV, Viçosa, 2006.

[4] Dolinsky, M., De Oliveira, R. M. da S. C., Rocha, G. de S., Barroso, S. G., Das Dores, S. M. C. e Azeredo, V. B. Efeitos do consumo de alimentos funcionais no perfil lipídico e estado nutricional de idosos, Internacional Journal of Cardiovascular Sciences, 28:400-408, 2015. DOI: $10.5935 / 2359-4802.20150059$.

[5] Marolli, A., Da Silva, J.A.G., Sawicki, S., Binelo, M.O., Scremin, A.H., Reginatto, D.C., Dornelles, E.F. e Lambrecht, D.M. A simulação da biomassa de aveia por elementos climáticos, nitrogênio e regulador de crescimento, Arquivo Brasileiro de Medicina Veterinária e Zootecnia, 70:535-544, 2018. DOI: 10.1590/1678-4162-9504.

[6] Medeiros, A. de S., Alves, F. I. dos S., Ferraz, R. L. de S., Campos, A. C., De Queiroz, M. M. F. e Magalhães, I. D. Crescimento e alocação de fitomassa do quiabeiro submetido à doses de nitrogênio e irrigação com água residuária, Revista Brasileira de Agricultura Irrigada, 12:2621-2631, 2018. DOI: 10.7127/rbai.v12n300775.

[7] Rosa, H. T., Walter, L. C., Streck, N. A. e Alberto, C. M. Métodos de soma térmica e datas de semeadura na determinação de filocrono de cultivares de trigo, Pesquisa Agropecuária Brasileira, 44:1374-1382, 2009. DOI: 10.1590/S0100-204X2009001100002.

[8] Silva, J. P., Rodrigues, B. A., Casado, J. C. S. and Rosa, S. S. R. F. Infusion Pump: Bond graph mathematical modeling for blood pressure control, IEEE Latin America Transactions, 16:1569-1573, 2018. DOI: 10.1109/TLA.2018.8444151. 